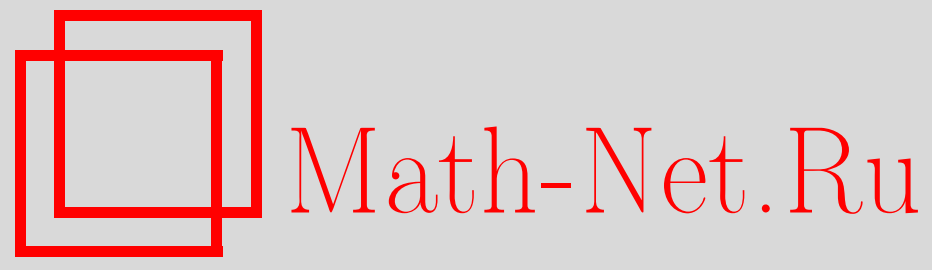

М. А. Нефедов, В. А. Салеев, Рождение бозонов Хиггса в реджевском пределе квантовой хромодинамики на БАК, Вестн. Сам. гос. техн. ун-та. Сер. Физ.-мат. науки, 2013, выпуск 2(), 250-258

DOI: https://doi.org/10.14498/vsgtu1174

Использование Общероссийского математического портала Math-Net.Ru подразумевает, что вы прочитали и согласны с пользовательским соглашением

http: //www . mathnet.ru/rus/agreement

Параметры загрузки:

IP : 54.89 .56 .158

26 апреля 2023 г., 11:59:10

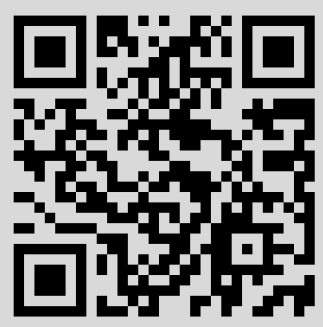


Вестн. Сам. гос. техн. ун-та. Сер. Физ.-мат. науки. 2013. № 2 (31). С. 250-258

УДК 539.1

\title{
РОЖДЕНИЕ БОЗОНОВ ХИГГСА В РЕДЖЕВСКОМ ПРЕДЕЛЕ КВАНТОВОЙ ХРОМОДИНАМИКИ НА БАК
}

\author{
М. А. Нефедов, В.А. Салеев \\ Самарский государственный университет, \\ Россия, 443011, Самара, ул. Академика Павлова, 1. \\ E-mails: nefedovma@gmail.com, saleev@samsu.ru
}

В теории реджезованных партонов Л. Н. Липатова рассматривается рождение скалярного бозона Хиггса Н Стандартной модели и псевдоскалярного бозона Хиггса А минимальной суперсимметричной Стандартной модели в процессах слияния реджезованных глюонов $(R R \rightarrow H, A)$ nри энергиях Большого адронного коллайдера. Расчеты выполнены в лидирующем приближении по константе сильного взаимодействия $\alpha_{s}$ с учетом вкладов от $t$ - $u$ b-кварков в петлевые интегралы. Рассчитаны спектры по поперечному импульсу и зависимость полных сечений рождения от масс бозонов. Полученные результать согласуются с предсказаниями, полученными ранее в коллинеарной партонной модели с учетом следующих поправок по $\alpha_{s}$ и эффектов суммирования вкладов, усиленных большими логарифмами, во всех порядках теории возмущений.

Ключевые слова: бозон Хиггса, Стандартная модель, минимальная суперсимметричная Стандартная модель, квантовая хромодинамика, теория реджезованных партонов.

Введение. Исследование процессов рождения бозонов Хиггса в Стандартной модели (CM) и минимальной суперсимметричной Стандартной модели (MCCM) на Большом адронном коллайдере (БАК) рассматривается как центральная задача физики высоких энергий текущего десятилетия. Обнаружение на БАК стандартного скалярного бозона Хиггса будет блестящим подтверждением СМ и очередным триумфом квантовой калибровочной теории поля. Другая важная проблема, которую ожидается решить в экспериментах на БАК, это получение ответа на вопрос о месте суперсимметричных обобщений СМ. Первые указания на существование скалярной частицы с массой около 125 ГэВ были получены в 2012 году Коллаборациями ATLAS [1] и CMS [2]. Превышение сигнала над фоном составляет около 4 стандартных отклонений, что позволяет надеяться на достоверность полученных результатов. В этой связи ещё более актуальным становится моделирование процессов рождения бозонов Хиггса на БАК в теории реджезованных партонов (ТРП), которая позволяет учесть нетривиальные эффекты, связанные с особенностями квантовой хромодинамики (КХД) при высоких и сверхвысоких энергиях.

В СМ основной вклад в сечения рождения хиггсовского бозона с массой порядка $100 \div 500$ ГэВ в протон-протонных взаимодейсвиях при энергии протонов в системе центра масс на БАК $\sqrt{s}=7 \div 14$ ТэВ дает процесс глюонглюонного слияния $g+g \rightarrow H$, идущий через кварковую петлю. Наибольший вклад в этот процесс дает петля $t$-кварка, т. к. вершина взаимодействия

Максим Александрович Нефедов, аспирант, каф. общей и теоретической физики. Владимир Анатольевич Салеев (д.ф.-м.н., проф.), профессор, каф. математического моделирования в механике. 
бозона Хиггса СМ пропорциональна массе кварка в петле:

$$
G_{H}=i \frac{m}{v}, \quad v=\frac{1}{\left(\sqrt{2} G_{F}\right)^{1 / 2}},
$$

где $G_{F}$ - константа Ферми. Величина $v \simeq 246$ ГэВ характеризует энергетический масштаб спонтанного нарушения электрослабой симметрии. Следующий по величине вклад дает процесс рождения глюонами хиггсовского бозона совместно с парой «кварк-антикварк», в основном с парой $b$-кварков $(b \bar{b} H)$, затем идут процессы ассоциативного рождения бозона Хиггса с тяжелыми калибровочными бозонами $(W H, Z H)$ и парой $t$-кварков $(t \bar{t} H)$.

В связи с экспериментальными условиями, предполагающими, что на начальном этапе поиска бозона Хиггса на БАК статистика будет недостаточной для измерения дифференциальных распределений, имеющиеся расчеты в коллинеарной партонной модели (KПМ) в основном ориентированы на определение полного сечения рождения бозона Хиггса в СМ, которое в настоящее время известно как в следующем за лидирующим приближении по $\alpha_{s}$ (СЛП) с учётом конечной массы топ кварка [5], так и в следующем за СЛП КПМ в пределе $m_{t} \rightarrow \infty[6]$. Для расчета распределений по поперечному импульсу $\left(p_{T}\right)$ используется широкий класс генераторов Монте-Карло [7] в сочетании с процедурой суммирования больших логарифмических поправок в КПМ [7-9]. Сочетание этих методов позволяет получать стабильные предсказания для $p_{T}$-распределений, которых достаточно для целей феноменологии СМ. Однако в феноменологии МССМ мы вынуждены иметь дело с большим числом свободных параметров и делать предсказания при их варьировании в широкой области. Это приводит к существенным неопределенностям при использовании упомянутых выше подходов.

В МССМ спонтанное нарушение электрослабой симметрии обеспечивается введением двух дублетов хиггсовских полей, что приводит к возникновению двух скалярных $(H$ и $h)$, одного псевдоскалярного $(A)$ и двух заряженных $\left(H^{ \pm}\right)$бозонов Хиггса. Генерация масс верхних и нижних фермионов в $S U(2)$-дублете обеспечивается разными дублетами хиггсовских полей с различными вакуумными средними для этих дублетов $v_{t}$ и $v_{b}$. В результате естественным параметром модели является отношение этих вакуумных средних:

$$
\operatorname{tg}(\beta)=\frac{v_{b}}{v_{t}} .
$$

Вершины взаимодействия псевдоскалярного бозона Хиггса МССМ с фермионами имеют вид

$$
G_{b}^{(A)}=\frac{m_{b}}{v} \operatorname{tg}(\beta) \gamma_{5}, \quad G_{t}^{(A)}=\frac{m_{t}}{v} \operatorname{ctg}(\beta) \gamma_{5}
$$

Экспериментальные данные [4] дают следующие ограничения на свободные параметры MCCM: $10<\operatorname{tg}(\beta)<30$ и $M_{A}>100$ ГэВ; при таких значениях параметров вклады петель $t$ - и $b$-кварков в амплитуду рождения бозона Хиггса $A$ будут иметь одинаковый порядок величины. С другой стороны, неколлинеарные поправки к петле $b$-кварка могут быть значительны, т. к. поперечные импульсы реджезованных глюонов имеют величину порядка массы $b$-кварка, но много меньше массы $t$-кварка. В КПМ последнее обстоятельство 
приводит к необходимости непростого учета старших поправок теории возмущений, в то время как в ТРП основная часть этих поправок учитывается уже в лидирующем приближении.

\section{1. Амплитуды рождения скалярного и псевдоскалярного бозонов Хиггса} в ТРП. При высоких энергиях основной вклад в инклюзивные сечения рождения частиц дают процессы в мультиреджевской кинематике. В этих условиях начальные $t$-канальные партоны реджезуются, что является фундаментальным свойством всех калибровочных теорий поля. В работах Л. Н. Липатова сформулирована ТРП как обобщение КХД [11]. Лагранжиан ТРП содержит поля реджезованных глюонов и кварков, а их взаимодействие с Янг-Миллсовскими глюонами и кварками ПМ осуществляется при помощи индуцированных вершин [12]. В рассматриваемом процессе важна вершина перехода реджезованного глюона в Янг-Миллсовский $R \rightarrow g$ :

$$
\underset{\text { Wunee }}{a: \pm}=\Gamma_{a b}^{ \pm \nu}(q)=i \delta_{a b} q^{2}\left(n^{ \pm}\right)^{\nu},
$$

где $n^{+^{\mu}}=2 P_{1}^{\mu} / \sqrt{S}, n^{-\mu}=2 P_{2}^{\mu} / \sqrt{S},\left(n^{+}\right)^{2}=\left(n^{-}\right)^{2}=0,\left(n^{+} n^{-}\right)=2,2\left(P_{1} P_{2}\right)=$ $=S, P_{1,2}-4$-импульсы сталкивающхся протонов.

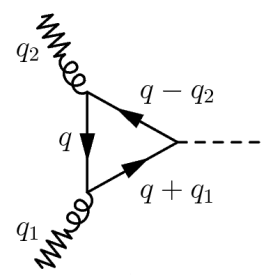

a

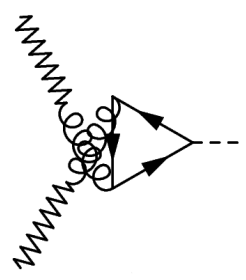

б

Рис. 1 приближении для процесса $R R \rightarrow H$ приведены на рис. 1.

Необходимо отметить, что для согласования правил Фейнмана в ТРП с правилами Фейнмана в стандартной КХД амплитуды в ТРП домножается на нормировочный множитель

$$
\mathcal{N}=\frac{x_{1} x_{2} S}{4 \sqrt{t_{1} t_{2}}},
$$

где $q_{1,2}=x_{1,2} P_{1,2}+q_{1,2 T}-4$-импульсы реджезованных партонов, $q_{T}=$ $=\left(0, \mathbf{q}_{T}, 0\right), t_{1,2}=\mathbf{q}_{1,2 T}^{2}$. В ТРП амплитуда рождения скалярного бозона Хиггса в слиянии двух реджезованых глюонов (упускаем константу связи и цветовую структуру) имеет вид

$$
\begin{aligned}
& \mathcal{A}_{S}\left(t_{1}, t_{2}, M_{T}, M_{H}\right)=\frac{(-1)}{(2 \pi)^{4}} \frac{x_{1} x_{2} P_{1 \mu} P_{2 \nu}}{\sqrt{t_{1}} \sqrt{t_{2}}} \times \\
& \times\left\{\int d^{4} q \frac{\operatorname{Tr}\left[\gamma^{\mu}(\hat{q}+m) \gamma^{\nu}\left(\hat{q}-\hat{q}_{2}+m\right)\left(\hat{q}+\hat{q}_{1}+m\right)\right]}{\left(q^{2}-m^{2}\right)\left(\left(q-q_{2}\right)^{2}-m^{2}\right)\left(\left(q+q_{1}\right)^{2}-m^{2}\right)}+\left(q_{1} \leftrightarrow q_{2}, \mu \leftrightarrow \nu\right)\right\},
\end{aligned}
$$

где $m$ - масса кварка, а $M_{H}$ - масса бозона Хиггса. Скалярные произведения, через которые выражается амплитуда, записываются в таком виде:

$$
\left(q_{1}+q_{2}\right)^{2}=M_{H}^{2}, \quad 2\left(q_{1} q_{2}\right)=M_{H}^{2}+t_{1}+t_{2}, \quad x_{1} x_{2} S=M_{H}^{2}+\mathbf{p}_{T}^{2}=M_{T}^{2},
$$

где $\mathbf{p}_{T}=\mathbf{q}_{1 T}+\mathbf{q}_{2 T}$. В рамках метода размерной регуляризации и процедуры Пассарино-Вельтмана [13] данная амплитуда может быть сведена к линейной комбинации базисных однопетлевых интегралов $B_{0}$ и $C_{0}$ с коэффициентами, зависящими от $t_{1}, t_{2}$. Выражения для этих коэффициентов являются 
весьма громоздкими, но они могут быть упрощены, если ввести новые функции:

$$
D_{ \pm}\left(t_{1}, t_{2}\right)=M_{H}^{4} \pm 2 M_{H}^{2}\left(t_{1}+t_{2}\right)+\left(t_{1}-t_{2}\right)^{2}
$$

Тогда выражение для амплитуды принимает вид

$$
\begin{aligned}
& \mathcal{A}_{S}=\frac{(-i) m M_{T}^{2}}{8^{3} \pi^{2} M_{H}^{4} D_{+}^{2} \sqrt{t_{1} t_{2}}}\left\{A \cdot C_{0}\left(M_{H}^{2},-t_{1},-t_{2}, m^{2}, m^{2}, m^{2}\right)+\right. \\
&+8 B \cdot B_{0}\left(M_{H}^{2}, m^{2}, m^{2}\right)-4 C\left(t_{1}, t_{2}\right) \cdot B_{0}\left(-t_{1}, m^{2}, m^{2}\right)- \\
&\left.-4 C\left(t_{2}, t_{1}\right) \cdot B_{0}\left(-t_{2}, m^{2}, m^{2}\right)+32 D\right\}
\end{aligned}
$$

где коэффициенты $A, B, C, D$ имеют следующий вид:

$$
\begin{aligned}
& A=M_{T}^{2}\left(D_{-}-D_{+}-4 M_{H}^{4}\right) \times \\
& \times\left(-8 M_{H}^{4}\left(3 D_{-}+5 D_{+}\right)-2 D_{-} D_{+}+3 D_{-}^{2}+64 m^{2} D_{+} M_{H}^{2}-D_{+}^{2}+48 M_{H}^{8}\right)+ \\
& +4 D_{+} M_{H}^{2}\left(-8 M_{H}^{4}\left(D_{-}+3 D_{+}\right)+2 D_{-} D_{+}+D_{-}^{2}+\right. \\
& \left.+64 m^{2} D_{+} M_{H}^{2}-3 D_{+}^{2}+16 M_{H}^{8}\right) \\
& B=M_{H}^{2}\left(M_{T}^{2}\left(24 M_{H}^{4}\left(D_{-}+D_{+}\right)+2 D_{-} D_{+}-3 D_{-}^{2}+D_{+}^{2}-48 M_{H}^{8}\right)-\right. \\
& \left.-4 D_{+} M_{H}^{2}\left(D_{-}+3 D_{+}-4 M_{H}^{4}\right)\right) \text {, } \\
& C\left(t_{1}, t_{2}\right)=\left(D_{-}-D_{+}+4 M_{H}^{2}\left(t_{2}-t_{1}\right)\right)\left(3 M_{T}^{2}\left(D_{-}-4 M_{H}^{4}\right)\left(M_{H}^{2}-\left(t_{2}-t_{1}\right)\right)+\right. \\
& \left.+D_{+}\left(M_{H}^{2}\left(5 M_{T}^{2}-4\left(t_{2}-t_{1}\right)\right)+4 M_{H}^{4}+3 M_{T}^{2}\left(t_{2}-t_{1}\right)\right)\right), \\
& D=M_{H}^{2} D_{+}\left(M_{T}^{2}\left(D_{-}-4 M_{H}^{4}\right)+D_{+}\left(4 M_{H}^{4}-M_{T}^{2}\right)\right) .
\end{aligned}
$$

Амплитуда (2) не имеет ультрафиолетовых расходимостей, сингулярные члены $\Delta_{\overline{M S}}$, входящие в определение функции $B_{0}$, сокращаются. На стадии численных расчетов мы используем представление функции $C_{0}$ через комплексные дилогарифмы $\mathrm{Li}_{2}(z)$, полученное в работе [14]. Квадрат модуля амплитуды рождения СМ Хиггса в ЛП ТРП с учетом вклада $b$-кварка и опущенных ранее цветовых множителей и констант связи имеет вид

$$
\overline{\left|\mathcal{A}_{S M}\right|^{2}}=\frac{\pi^{2}}{\sqrt{2}} G_{F} \alpha_{s}^{2}\left|m_{t} \mathcal{A}_{S}\left(m=m_{t}\right)+m_{b} \mathcal{A}_{S}\left(m=m_{t}\right)\right|^{2} .
$$

После перехода к пределу $t_{1,2} \rightarrow 0$ в (3) и усреднения по углу между поперечными импульсами партонов квадрат модуля амплитуды в ТРП переходит в соответствующий квадрат модуля амплитуды в КПМ [15]:

$$
\overline{\left|\mathcal{A}_{S}^{C P M}\right|^{2}}=\frac{\alpha_{s}^{2} G_{F} M_{H}^{4} \sqrt{2}}{256 \pi^{2}}|\tau(1+(1-\tau) f(\tau))|^{2},
$$

где

$$
f(\tau)=-\frac{M_{H}^{2}}{2} C_{0}\left(M_{H}^{2}, 0,0, \frac{M_{H}^{2} \tau}{4}, \frac{M_{H}^{2} \tau}{4}, \frac{M_{H}^{2} \tau}{4}\right), \quad \tau=\frac{4 m^{2}}{M_{H}^{2}}
$$


Рассмотрим рождение псевдоскалярного бозона Хиггса МССМ. Амплитуда записывается аналогично (1):

$$
\begin{gathered}
\mathcal{A}_{P S}=\frac{(-1)}{(2 \pi)^{4}} \frac{x_{1} x_{2} P_{1 \mu} P_{2 \nu}}{\sqrt{t_{1}} \sqrt{t_{2}}} \mathcal{A}_{\mathcal{P} \mathcal{S}}^{\mu \nu}\left(-t_{1},-t_{2}, M_{A}^{2}, m\right), \\
\mathcal{A}_{\mathcal{P S}}^{\mu \nu}\left(-t_{1},-t_{2}, M_{A}^{2}, m\right)=\int d^{4} q \frac{\operatorname{Tr}\left[\gamma^{\mu}(\hat{q}+m) \gamma^{\nu}\left(\hat{q}-\hat{q}_{2}+m\right) \gamma_{5}\left(\hat{q}+\hat{q}_{1}+m\right)\right]}{\left(q^{2}-m^{2}\right)\left(\left(q-q_{2}\right)^{2}-m^{2}\right)\left(\left(q+q_{1}\right)^{2}-m^{2}\right)}+ \\
+\left(q_{1} \leftrightarrow q_{2}, \mu \leftrightarrow \nu\right) .
\end{gathered}
$$

Можно показать, что квадрат модуля амплитуды рождения бозона Хиггса $A$ в ЛП ТРП приводится к виду

$$
\begin{aligned}
\overline{\left|\mathcal{A}_{A}\right|^{2}}=\frac{2 \alpha_{s}^{2} M_{T}^{4}}{(8 \pi v)^{2}} \sin ^{2}(\phi) \mid m_{b}^{2} C_{0}( & \left.M_{A}^{2},-t_{1},-t_{2}, m_{b}^{2}, m_{b}^{2}, m_{b}^{2}\right) \operatorname{tg}(\beta)+ \\
& +\left.m_{t}^{2} C_{0}\left(M_{A}^{2},-t_{1},-t_{2}, m_{t}^{2}, m_{t}^{2}, m_{t}^{2}\right) \operatorname{ctg}(\beta)\right|^{2}
\end{aligned}
$$

где $\phi-$ угол между векторами $\mathbf{q}_{1 T}$ и $\mathbf{q}_{2 T}$. В коллинеарном пределе получаем

$$
\overline{\left|\mathcal{A}_{A}^{C P M}\right|^{2}}=\frac{\alpha_{s}^{2} M_{A}^{4}}{4(8 \pi v)^{2}}\left|\tau_{b} f\left(\tau_{b}\right) \operatorname{tg}(\beta)+\tau_{t} f\left(\tau_{t}\right) \operatorname{ctg}(\beta)\right|^{2}
$$

где $\tau_{t, b}=4 m_{t, b}^{2} / M_{H}^{2}$, что совпадает с известным выражением в КПМ [16].

2. Сечения рождения бозона Хиггса СМ на БАК. Дифференциальное по поперечному импульсу $\left(p_{T}\right)$ и быстроте $(y)$ сечение рождения бозона Хиггса в слиянии реджезованных глюонов согласно теореме факторизации в ТРП записывается в виде [17]:

$$
\frac{d^{2} \sigma}{d y d p_{T}}=\frac{p_{T}}{M_{T}^{4}} \int_{0}^{\infty} d t_{1} \int_{0}^{2 \pi} d \phi_{1} \Phi_{g}^{p}\left(x_{1}, t_{1}, \mu_{F}\right) \Phi_{g}^{p}\left(x_{2}, t_{2}, \mu_{F}\right) \overline{\left|\mathcal{A}_{\mathcal{S M}}\right|^{2}}
$$

где

$$
x_{1,2}=\frac{M_{T}}{\sqrt{S}} e^{ \pm y}, \quad M_{T}=\sqrt{M_{H}^{2}+p_{T}^{2}}, \quad t_{2}=p_{T}^{2}+t_{1}-2 p_{T} \sqrt{t_{1}} \cos \left(\phi_{1}\right)
$$

$\phi_{1}$ - угол между $\mathbf{q}_{1 T}$ и $\mathbf{p}_{T}$, а $\Phi\left(x, t, \mu_{f}^{2}\right)$ - глюонная неинтегрированная партонная функция распределения (НПФР). В наших расчетах мы будем использовать глюонную НПФР, полученную в работах Кимбера, Мартина и Рыскина [19], которая соответствует коллинеарной ПФР MRST2002 [18]. В ТРП мы учитываем лидирующие логарифмические поправки вида $\ln \left(\mu / \Lambda_{Q C D}\right)$ и $\ln (1 / x)$, в то же время из расчётов в СЛП КПМ известно, что нелогарифмические поправки также дают большой вклад в сечение. Эти вклады обусловлены петлевыми глюонными поправками к вершине рождения Хиггса и могут быть эффективно учтены при помощи умножения сечения на $K$-фактор:

$$
K=\exp \left[\frac{\pi}{2} C_{A} \alpha_{s}\left(\mu_{R}\right)\right]
$$


где $C_{A}=N_{c}=3$. Данный $K$-фактор получается из аналитического продолжения формфактора Судакова во времениподобную область переданных импульсов (см., например, [20] и имеющиеся там ссылки).

При вычислении сечений мы используем следующий выбор масштабов перенормировки и факторизации: $\mu_{R}=\mu_{F}=\xi M_{T}$, где для центральной кривой на графиках соответствует прямая $\xi=1$, а коридор теоретической неопределённости вносимой выбором масштаба, строится путём варьирования $1 / 2<\xi<2$.

На рис. 2 приведено сравнение наших предсказаний (сплошная гистограмма и сплошная заливка) для $p_{T}$-спектра бозонов Хиггса при $\sqrt{s}=7$ ТэВ и $M_{H}=120$ ГэВ с учетом вклада $b$-кварка с результатами СЛП КПМ методом Монте-Карло, взятыми из работы [7]. Полоса неопределенности с вертикальной штриховкой соответствует результату генератора Монте-Карло Pythia c программным кодом POWHEG [10]. Полоса неопределенности с горизонтальной штриховкой показывает результат расчета в СЛП КПМ в программе НqТ [9].

Рис. 2. показывает, что расчет в ЛП ТРП удовлетворительно воспроизводит результаты СЛП КПМ, особенно если принять во внимание степень неопределенности последних. Результат ЛП ТРП менее устойчив по отношению к варьированию масштабов перенормировки и факторизации, что, впрочем, характерно для расчетов в лидирующем приближении.

Предсказания ЛП ТРП и СЛП КПМ для зависимости полного сечения рождения бозона Хиггса СМ от его массы в наиболее интересной для эксперимента области $120<M_{H}<130$ ГэВ отличаются не более чем на $25 \%$, что является хорошим аргументом в пользу применения расчетов в ЛП ТРП для оценки различных дифференциальных сечений. В частности мы можем сделать предсказание для $p_{T}$-спектра бозона Хиггса с массой 126 ГэВ при $\sqrt{s}=14$ ТэВ, который может быть измерен после модернизации БАК до этой энергии (рис. 3).

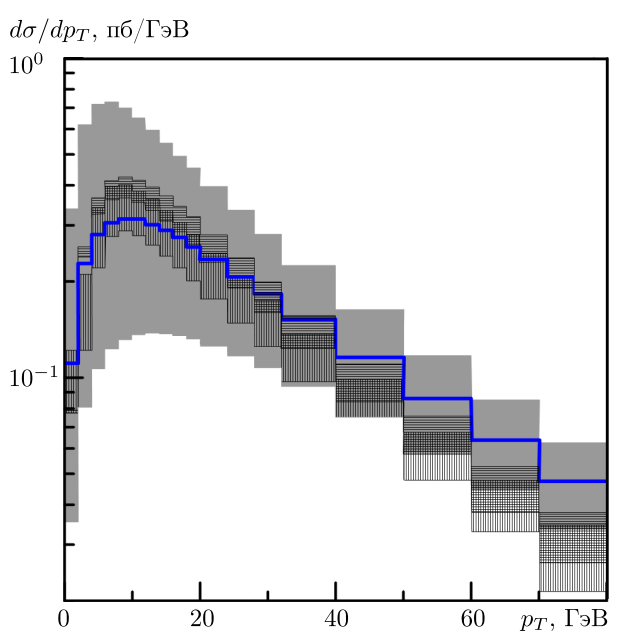

Рис. 2

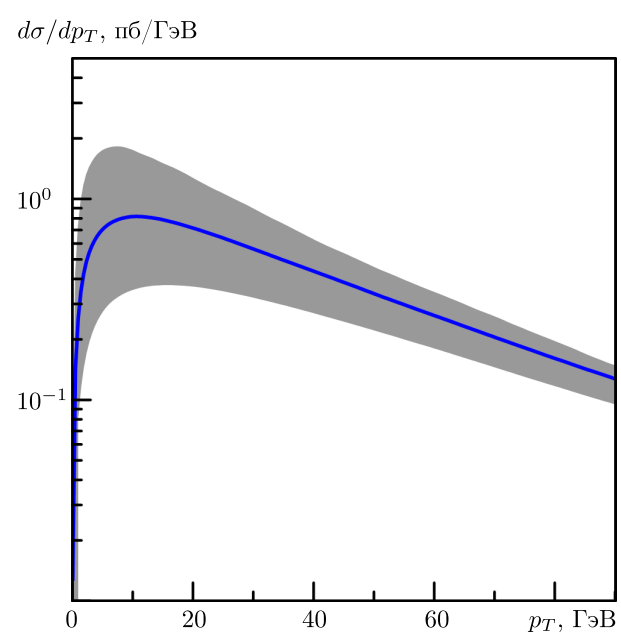

Рис. 3

3. Сечения рождения псевдоскалярного бозона Хиггса МССМ. На рис. 4 приведено сравнение наших предсказаний для полного сечения рождения бозона Хиггса MCСМ $A$ в ЛП ТРП при $\operatorname{tg}(\beta)=3$ (кривая 1) и $\operatorname{tg}(\beta)=30$ 


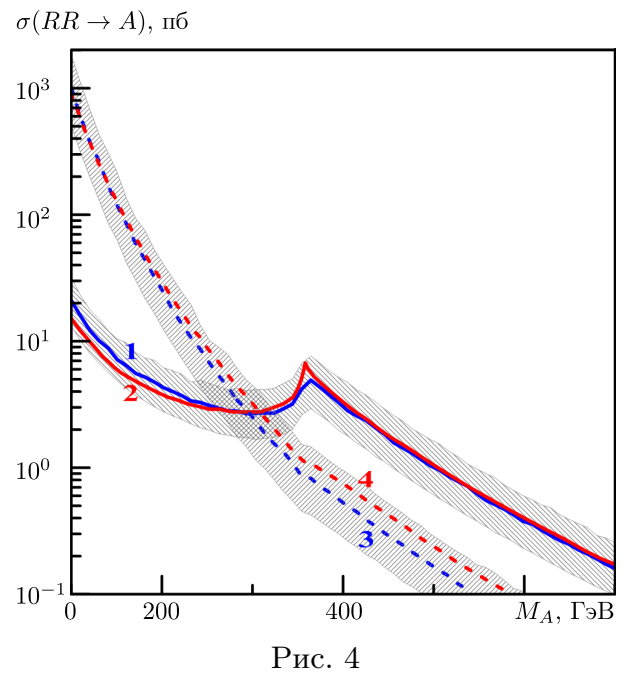

(кривая 3) с соответствующими результатами, полученными в СЛП КПМ [16] (кривые 2 и 4). Видно, что при $\operatorname{tg}(\beta)=3$ ЛП ТРП хорошо воспроизводит результат СЛП КПМ практически для всех значений массы бозона, в то время как для $\operatorname{tg}(\beta)=30$ при больших значениях массы наш подход предсказывает несколько меньшее сечение, чем СЛП КПМ. Однако кривая КПМ лежит в пределах полосы теоретической неопределенности наших расчетов, что позволяет надеяться на разумное согласие предсказаний и для спектров по поперечному импульсу с результатами КПМ.

Результаты расчетов дифференциального эффективного сечения рождения псевдоскалярного бозона Хиггса МССМ в процессе слияния реджезованных глюонов в ЛП ТРП для $\sqrt{s}=14$ ТэВ при значениях $M_{A}=126$, 200, 500, 1000 ГэВ приведены на рис. 5 (а, б, в, г, соответственно). Сплошная кривая соответствует значению $\operatorname{tg}(\beta)=3$, а штриховая кривая $-\operatorname{tg}(\beta)=30$. Из рисунков видно, что спектры при большом и малом значениях $\operatorname{tg}(\beta)$ имеют разный наклон. В случае измерения данных спектров на эксперименте этот факт можно использовать для более точного определения значения этого параметра.
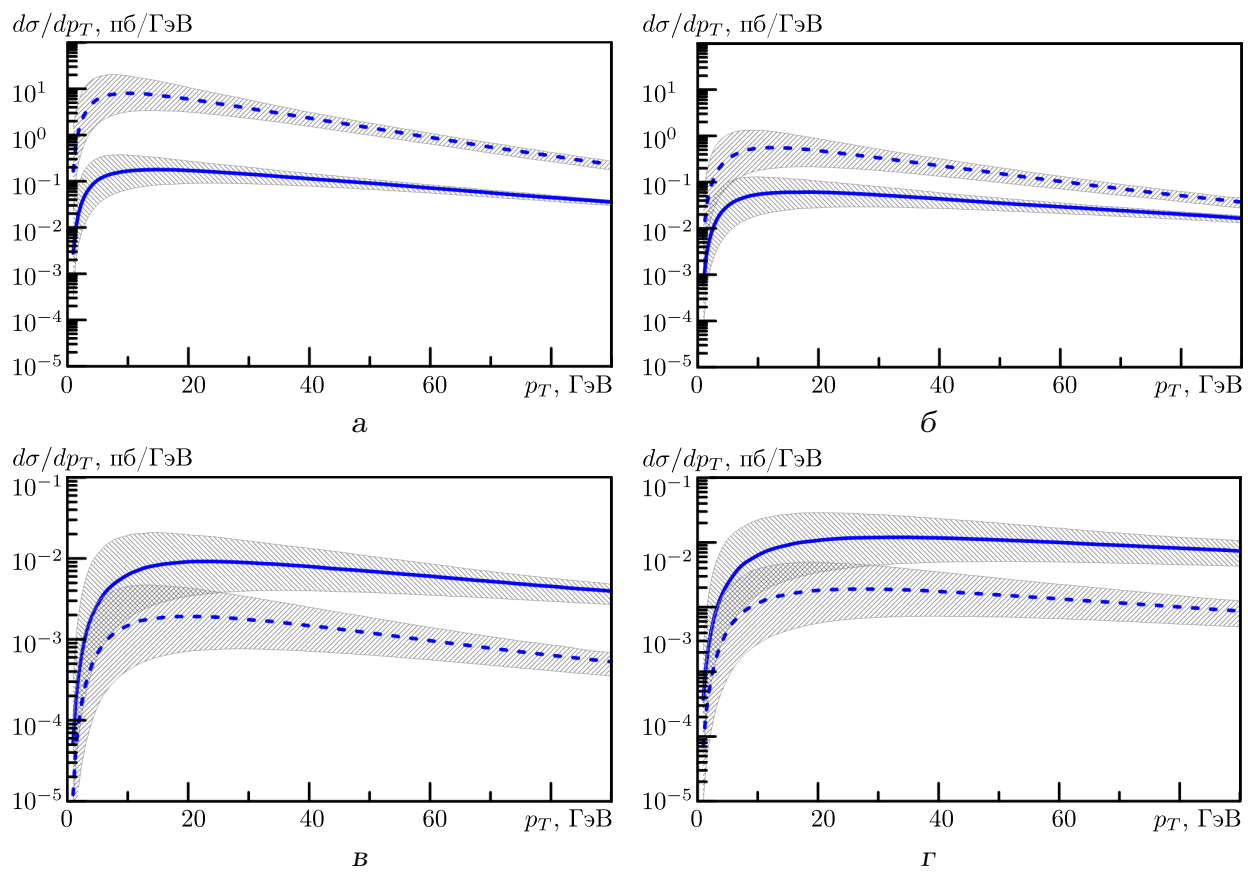

Рис. 5 
Также можно заключить, что при увеличении $M_{A}$ сечение, соответствующее $\operatorname{tg}(\beta)=30$, становится меньше, чем сечение для $\operatorname{tg}(\beta)=3$. Это связано с влиянием интерференции $t$ и $b$-кварковых вкладов, которая становится деструктивной при $M_{A} \gg m_{t, b}$ в силу свойств функции $C_{0}$.

Заключение. Эксперименты на БАК в тэвной области энергий показывают, что доминирующую роль в процессах рождения частиц в центральной области по быстроте играют подпроцессы в мультиреджевской кинематике, для описания которых необходимо использовать ТРП вместо КПМ. Результаты этой работы, а также наших расчетов спектров чармониев [21], $b$-кварковых струй [22], адронных струй и прямых фотонов [23] подтверждают это наблюдение.

Работа выполнена при поддержке РФФИ (проекты №№ 11-02-00769-а, 12-02-31701-мол-а).

\section{БИБЛИОГРАФИЧЕСКИЙ СПИСОК}

1. ATLAS Collaboration, G. Aad, et. al., "Observation of a new particle in the search for the Standard Model Higgs boson with the ATLAS detector at the LHC" // Phys. Lett. B., 2012. Vol. 716, no. 1. Pp. 1-29, arXiv: 1207.7214 [hep-ex].

2. CMS Collaboration, S. Chatrchyan, et. al., "Observation of a new boson at a mass of 125 GeV with the CMS experiment at the LHC" // Phys. Lett. B., 2012. Vol.716, no. 1. Pp. 3061, arXiv: 1207.7235 [hep-ex].

3. J. Baglio, A. Djouadi, R. M. Godbole, "The apparent excess in the Higgs to di-photon rate at the LHC: New Physics or QCD uncertainties?" // Phys. Lett. B, 2012. Vol.716, no. 1. Pp. 203-207, arXiv: 1207.1451 [hep-ph].

4. ATLAS Collaboration, G. Aad, et. al., "Search for neutral MSSM Higgs bosons decaying to $\tau^{+} \tau^{-}$pairs in proton-proton collisions at $\sqrt{s}=7 \mathrm{TeV}$ with the ATLAS detector" // Phys. Lett. B, 2011. Vol. 705, no. 3. Pp. 174-192, arXiv: 1107.5003 [hep-ex].

5. M. Spira, A. Djouadi, D. Graudenz, P. M. Zerwas, "Higgs boson production at the LHC" // Nucl. Phys. B., 1995. Vol. 453, no.1-2. Pp. 17-82, arXiv: hep-ph/9504378.

6. C. Anastasiou, K. Melnikov, "Higgs boson production at hadron colliders in NNLO QCD" // Nucl. Phys. B., 2002. no. 1-2. Pp. 220-256, arXiv: hep-ph/0207004.

7. LHC Higgs Cross Section Working Group, S. Dittmaier, et. al., Handbook of LHC Higgs Cross Sections: 2. Differential Distributions: to be submitted to CERN Report, 2012. 275 pp., arXiv: hep-ph/1201.3084.

8. Yu. L. Dokshitzer, D. I. Dyakonov, S. I. Troyan, "Hard processes in quantum chromodynamics" // Phys. Rep., 1980. Vol. 58, no. 5. Pp. 269-395.

9. G. Bozzi, S. Catani, D. de Florian, M. Grazzini, "The $q_{T}$ spectrum of the Higgs boson at the LHC in QCD perturbation theory" // Phys. Lett. B, 2003. Vol.564, no. 1-2. Pp. 65-72, arXiv: hep-ph/0302104.

10. S. Frixione, P. Nason, C. Oleari, "Matching NLO QCD computations with parton shower simulations: the POWHEG method" // JHEP, 2007. Vol. 2007, no. 11, 070.

11. L. N. Lipatov, "Gauge invariant effective action for high energy processes in QCD" // Nucl. Phys. B., 1995. Vol. 452, no.1-2. Pp. 369-397, arXiv: hep-ph/9502308.

12. E. N. Antonov, L. N. Lipatov, E. A. Kuraev, I. O. Cherednikov, "Feynman rules for effective Regge action" // Nucl. Phys. B., 2005. Vol.721, no.1-3. Pp. 111-135, arXiv: hep-ph/0411185.

13. G. Passarino, M. Veltman, "One-loop corrections for $e^{+} e^{-}$annihilation into $\mu^{+} \mu^{-}$in the Weinberg model" // Nucl. Phys. B., 1979. Vol. 160, no. 1. Pp. 151-207.

14. О. Л. Веретин, О. В. Теряев, Аксиальная аномалия при произвольных виртуальностях: Препринт ОИЯИ № Р2-94-483. Дубна: ОИЯИ, 1994. 12 с. [O. L. Veretin, O. V. Teryaev, Axial anomaly at the arbitrary external momenta: JINR Preprint P2-94-483. Dubna: JINR, 1994. 12 pp.] 
15. A. Djouadi, "The anatomy of electroweak symmetry breaking: Tome I: The Higgs boson in the Standard Model" // Phys. Rept., 2008. Vol.457, no.1-4. Pp. 1-216, arXiv: hep-ph/0503172.

16. A. Djouadi, "The anatomy of electroweak symmetry breaking Tome II: The Higgs bosons in the Minimal Supersymmetric Model" // Phys. Rept., 2008. Vol.459, no. 1-6. Pp. 1-241, arXiv: hep-ph/0503173.

17. B. L. Ioffe, V. S. Fadin, L. N. Lipatov, Quantum Chromodynamics, Perturbative and Nonperturbative Aspects. Cambridge, UK: Cambridge University Press, 2010. 596 pp.

18. A. D. Martin, R. G. Roberts, W. J. Stirling, R. S. Thorne, "NNLO global parton analysis" // Phys. Lett. B, 2002. Vol. 531, no. 3-4. Pp. 216-224, arXiv: hep-ph/0201127.

19. M. A. Kimber, A. D. Martin, M. G. Ryskin, "Unintegrated parton distributions" // Phys. Rev. D, 2001. Vol. 63, no. 11, 114027. 10 pp., arXiv: hep-ph/0101348.

20. G. Watt, A. D. Martin, M. G. Ryskin, "Unintegrated parton distributions and electroweak boson production at hadron colliders" // Phys. Rev. D, 2004. Vol. 70, no. 1, 014012. 9 pp., arXiv: hep-ph/0309096.

21. V. A. Saleev, M. A. Nefedov, A. V. Shipilova, "Prompt $J / \psi$ production in the Regge limit of QCD: From the Tevatron to the LHC" // Phys. Rev. D, 2012. Vol. 85, no. 7, 074013. 9 pp., arXiv: 1201.3464 [hep-ph].

22. V. A. Saleev, A. V. Shipilova, "Inclusive $b$-jet and $b \bar{b}$-dijet production at the LHC via Reggeized gluons" // Phys. Rev. D, 2012. Vol. 86, no. 3, 034032. 9 pp., arXiv: 1201.4640 [hep-ph].

23. B. A. Kniehl, V. A. Saleev, A. V. Shipilova, E. V. Yatsenko, "Single jet and prompt-photon inclusive production with multi-Regge kinematics: From Tevatron to LHC" // Phys. Rev. D, 2011. Vol. 84, no. 7, 074017. 8 pp.

Поступила в редакцию $15 / \mathrm{XI} / 2012$;

в окончательном варианте - 27/III/2013.

\title{
MSC: 81V05, 81T60
}

\section{HIGGS BOSON PRODUCTION AT THE LHC IN THE FRAMEWORK OF REGGE LIMIT OF QUANTUM CHROMODYNAMICS}

\section{A. Nefedov, V.A.Saleev}

Samara State University,

1, Academician Pavlov st., Samara, 443011, Russia.

E-mails: nefedovma@gmail.com, saleev@samsu.ru

\begin{abstract}
In the framework of the L. N. Lipatov Reggeized Partons Theory we consider production of scalar Higgs boson $H$ of the Standard Model and pseudoscalar Higgs boson A of the Minimal Supersymmetric Standard Model in the Reggeized gluon fusion at the energy range of the Large Hadron Collider. Working in the leading order approximation in the strong coupling constant $\alpha_{s}$ we take into account contributions from $t$ - and $b$-quark in the loop integrals. We have calculated transverse momentum boson spectra and total cross sections as a function of boson mass. The obtained results agree with predictions which have been obtained earlier in the collinear Parton Model with the next to leading order in $\alpha_{s}$ corrections and all-order resummation of large logarithmical corrections.
\end{abstract}

Key words: Higgs boson, Standard Model, Minimal Supersymmetric Standard Model, Quantum Chromodynamics, Theory of Reggeized Partons.

Original article submitted 15/XI/2012; revision submitted $27 / \mathrm{III} / 2013$.

Maxim A. Nefedov, Postgraduate Student, Dept. of General and Theoretical Physics.. Vladimir A. Saleev (Dr. Sci. (Phys. \& Math.)), Professor, Dept. of Mathematical Modeling in Mechanics.. 